\title{
A FINITE-ENERGY BOUND ON THE APPROACH OF A DIFFUSION TO THE ZEROS OF ITS DENSITY
}

\author{
TIMOTHY C. WALLSTROM
}

(Communicated by George C. Papanicolaou)

\begin{abstract}
Since the drift coefficients of a finite-energy diffusion are undefined on the nodes, or zeros of the density, an important problem has been to show that the sample paths stay away from the nodes. In this paper it is shown that if the drift is locally a gradient and smooth on the complement of the nodes, and if the density is smooth, then the closest approach to the nodes can be bounded solely in terms of the time-integrated energy.
\end{abstract}

If $\xi(t)$ is a diffusion process with density $\rho$, forward drift $b$, and backward drift $b_{*}$ (to be defined below), then $\xi$ is said to have finite energy if, for any finite interval $[0, T]$,

$$
\int_{0}^{T} \mathbf{E}_{\frac{1}{4}}\left[\left(b^{2}(\xi(t), t)+b_{*}^{2}(\xi(t), t)\right)\right] d t<\infty,
$$

where $\mathbf{E}$ is the expectation. This criterion permits the drifts to diverge as $\rho^{-1 / 2}$ near the zeros of the density, or "nodes". Diffusions with singular drifts of this type arise naturally when one tries to treat the time-forward and time-backward processes on an equal footing. In particular, these drifts arise in the theory of stochastic mechanics, which is an interpretation of the time-reversal invariant equations of quantum mechanics in terms of diffusion theory [5].

Because of their severe drift singularities, finite-energy diffusions are not amenable to construction by the usual methods. In recent years, however, Carlen [2,3], Nelson [4], and Zheng [7] have all given methods for constructing such diffusions, subject to various regularity assumptions in addition to (1). Furthermore, it has been shown, under the additional assumptions of continuity of the density and of the drifts away from the nodes, that the sample paths of a finite-energy diffusion never reach the nodes $[1,4,7,8]$.

In this paper we show that if one also assumes that the drift is a gradient, a condition which holds automatically in stochastic mechanics, the closest approach of the paths of the diffusion can be bounded in terms of the timeintegrated energy. This is an improvement over the best previously known

Received by the editors June 14, 1988 and, in revised form, January 30, 1989.

1980 Mathematics Subject Classification (1985 Revision). Primary 60G17, 81C20.

Key words and phrases. Finite-energy diffusions, singular diffusions, nodes, stochastic mechanics. 
results, in which potentially divergent integrals of the derivatives of $b$ and $b_{*}$ also enter into the bound. The present result is particularly satisfying in that it bounds the nodal approach in terms of the same quantity whose finiteness was assumed in order to construct the diffusion.

The proof given will carry over to any Riemannian manifold $M$, but we restrict ourselves to $\mathbb{R}^{n}$ in order to simplify notation. Let $\xi(t)$ be a diffusion process on $\mathbb{R}^{n}$, with density $\rho$, forward drift $b$, backward drift $b_{*}$, and covariance equal to the metric tensor. The backward drift $b_{*}(x, t)$ is defined as $\lim _{d t \downarrow 0} \mathbf{E}^{x, t}\left[d_{*} \xi(t) / d t\right]$, where $\mathbf{E}^{x, t}$ is the conditional expectation given that $\xi(t)=x$, and $d_{*} \xi(t)=\xi(t)-\xi(t-d t)$. It is often useful to introduce quantities whose time-reversal properties are transparent; accordingly, we define the osmotic and current velocities $u$ and $v$ by $u=\frac{1}{2}\left(b-b_{*}\right)$ and $v=\frac{1}{2}\left(b+b_{*}\right)$. This implies that $b=v+u$ and $b_{*}=v-u$. It can be shown that $u=\nabla R$, where $R$ is defined by $\rho=e^{2 R}$ and that $v$ is the current velocity in the continuity equation for the density,

$$
\frac{\partial \rho}{\partial t}=-\nabla \cdot(v \rho)
$$

(In stochastic mechanics, this equation can be derived from the Schrödinger equation for $\psi$, with the identification $\rho=|\psi|^{2}$ and $v=\nabla(\operatorname{Im} \log \psi)$.) We let $\mathscr{P}_{t}$ and $\mathscr{F}_{t}$ denote the forward and backward filtrations, respectively; these are generated by the $\xi(s)$ with $s \leq t$ and $s \geq t$, respectively. This is the standard cast of characters in the theory of finite-energy diffusions; see [5] for more details.

We can describe the process $\xi$ by either a forward or backward stochastic differential equation:

$$
\begin{aligned}
& d \xi(t)=b(\xi(t), t) d t+d w, \text { or } \\
& d_{*} \xi(t)=b_{*}(\xi(t), t) d t+d_{*} w_{*},
\end{aligned}
$$

where $w\left(w_{*}\right)$ is a forward (backward) Wiener process, and where backward increments are defined (formally) by

$$
d_{*} F(t)=F(t)-F(t-d t)
$$

Let $A$ be defined by

$$
A=\frac{1}{2} \int_{0}^{T} \int_{M}\left(u^{2}+v^{2}\right) \rho d x d t .
$$

This is the same as (1), so $\xi$ is a finite energy diffusion if $A<\infty$.

Our proof is closely patterned after that in [4], in which Nelson introduced the beautiful idea of considering the process $R(\xi(t), t)$. This procedure leads to quantities $\Delta R(\xi(t), t)$ and $\Delta S(\xi(t), t)$, whose time integrals cannot immediately be bounded in terms of $A$. Note, however, that for any smooth $F(x, t)$, the stochastic process $F(\xi(t), t)$ satisfies the following stochastic differential 
equations:

$$
\begin{aligned}
d F & =\frac{\partial F}{\partial t} d t+b \cdot \nabla F d t+\nabla F \cdot d w+\frac{1}{2} \Delta F d t \\
d_{*} F & =\frac{\partial F}{\partial t} d t+b_{*} \cdot \nabla F d t+\nabla F \cdot d_{*} w_{*}-\frac{1}{2} \Delta F d t
\end{aligned}
$$

Subtracting the second equation from the first, and recalling that $b-b_{*}=2 u$, we obtain

$$
\Delta F d t=d F-d_{*} F-2 u \cdot \nabla F d t-\nabla F \cdot d w+\nabla F \cdot d_{*} w_{*} .
$$

The $d F-d_{*} F$ term will disappear when we integrate, and if $F$ is $R$ or $S$, the remaining terms can be bounded by $A$. In this way, we will bound the time integrals of $\Delta R(\xi(s), s)$ and $\Delta S(\xi(s), s)$ in terms of $A$.

In order to apply Itô's lemma to the processes $R(\xi(t), t)$ and $S(\xi(t), t)$, we must first show that the process $\xi(t)$ never reaches the nodes. In [4], Nelson proved this for finite-energy diffusions on a compact manifold. His result can easily be generalized to noncompact manifolds. Consider the process stopped upon exiting some sphere

$$
V_{d}=\{x:|x| \leq d\} .
$$

Then the methods of [4] suffice to show that the particle never reaches the nodes before exiting $V_{d}$ (see also [8]). We also know that, for a finite-energy diffusion, one can choose $d$ large enough that the probability of $\xi(t)$ exiting $V_{d}$ is arbitrarily small. (Simply bound each term in the stochastic integral for $\xi(t)$ separately.) Thus, the probability of the sample paths reaching the nodes is zero.

Theorem 1. Assume that $A=\frac{1}{2} \int_{0}^{T} \int_{M}\left(u^{2}+v^{2}\right) \rho d x d t<\infty$, and that $v$ is a local gradient: $v=\nabla S$. Assume also that $u$ and $v$ are smooth away from the nodal set. Then, given $\varepsilon>0$, there exists $\delta>0$, depending only on $A$ and the initial condition $\rho(x, 0)=\rho^{0}(x)$, such that

$$
\operatorname{Pr}\left\{\inf _{t \in[0, T]} \rho(\xi(t), t)>\delta\right\} \geq 1-\varepsilon .
$$

Proof. Since $\int \rho^{0}(x) d x=1$, there exists $\delta_{1}>0$ such that if $U=\left\{x: \rho^{0}(x) \leq\right.$ $\left.\delta_{1}\right\}, \int_{U} \rho^{0}(x) d x \leq \varepsilon / 2$. From this we infer the existence of $\lambda_{1}$ such that

$$
\operatorname{Pr}\left\{R(\xi(0), 0) \leq-\lambda_{1}\right\} \leq \varepsilon / 2,
$$

where $R$ is defined as $\frac{1}{2} \log \rho$.

Now consider the process $R(\xi(t), t)$. By the Itô calculus,

$$
d R=\frac{\partial R}{\partial t} d t+\nabla R \cdot d \xi+\frac{1}{2} \Delta R d t .
$$

Taking the gradient of the continuity equation and dividing by $\rho$, we obtain

$$
\frac{\partial R}{\partial t}=-\nabla R \cdot \nabla S-\frac{1}{2} \Delta S
$$


When substituted into the expression for $d R$, we will obtain terms in $\Delta R$ and $\Delta S$. Using equation (3) to substitute for $\Delta R$ and $\Delta S$, we obtain

$$
\begin{aligned}
d R= & \frac{1}{2}\left(d R-d_{*} R\right)-\frac{1}{2}\left(d S-d_{*} S\right)+(-u \cdot \nabla R+u \cdot \nabla S) d t \\
& -\frac{1}{2}\left(\nabla R \cdot d w-\nabla R \cdot d_{*} w_{*}\right)+\frac{1}{2}\left(\nabla S \cdot d w-\nabla S \cdot d_{*} w_{*}\right) \\
& +u \cdot v d t+b \cdot \nabla R d t+\nabla R \cdot d w .
\end{aligned}
$$

This is a local relation, so $S$ need not be globally defined. The time integrals of $\left(d R-d_{*} R\right)$ and $\left(d S-d_{*} S\right)$ vanish, and we find that

$$
R(\xi(t), t)-R(\xi(0), 0)=\int_{0}^{t} u \cdot v d s+\frac{1}{2} \int_{0}^{t} b \cdot d w-\frac{1}{2} \int_{0}^{t} b_{*} \cdot d_{*} w_{*} \cdot
$$

Let $X(t)$ be defined as $R(\xi(t), t)-R(\xi(0), 0)$. Then $X(t)$ is the sum of three integrals, which we label consecutively as $X_{i}(t)$, for $i=1,2,3$.

By Chebyshev's inequality,

$$
\operatorname{Pr}\left\{\sup _{t \in[0, T]}\left|X_{1}(t)\right| \geq \lambda\right\} \leq \frac{\int_{0}^{T} \int \rho|u \cdot v| d x d t}{\lambda} \leq \frac{A}{\lambda} .
$$

Since $X_{2}(t)$ is a $\mathscr{P}_{t}$-martingale, $X_{2}^{2}(t)$ is a nonnegative $\mathscr{P}_{t}$-submartingale, and by Doob's submartingale inequality,

$$
\operatorname{Pr}\left\{\sup _{t \in[0, T]}\left|X_{2}(t)\right| \geq \lambda\right\} \leq \frac{\int_{0}^{T} \int\left|\frac{1}{2} b\right|^{2} \rho d x d t}{\lambda^{2}} \leq \frac{A}{\lambda^{2}} .
$$

A similar estimate holds for $X_{3}(t)$. Therefore,

$$
\operatorname{Pr}\left\{\sup _{t \in[0, T]}|R(\xi(t), t)-R(\xi(0), 0)| \geq \lambda\right\} \leq\left(\frac{1}{\lambda}+\frac{2}{\lambda^{2}}\right) A .
$$

Note that as $\lambda \rightarrow \infty$, the bound will go as $A / \lambda$.

Choose $\lambda_{2}>0$ such that $\left(1 / \lambda_{2}+2 / \lambda_{2}^{2}\right) A \leq \varepsilon / 2$. Combining this with our previous estimate, we find that

$$
\operatorname{Pr}\left\{\inf _{t \in[0, T]} R(\xi(t), t) \leq-\lambda_{1}-\lambda_{2}\right\} \leq \varepsilon,
$$

which implies that

$$
\operatorname{Pr}\left\{\inf _{t \in[0, T]} \rho(\xi(t), t) \leq e^{-2\left(\lambda_{1}+\lambda_{2}\right)}\right\} \leq \varepsilon .
$$

Remarks. (1) If the process is stationary, then $\partial R / \partial t=0$, and $\Delta S$ does not enter into the calculation of $d R$. In this case, therefore, it is not necessary to assume that $v$ is a gradient. Note also that if $u \cdot v=0$, and in particular, if $v=0$, then $X_{1}(t)$ vanishes, and the bound falls off as $1 / \lambda^{2}$ instead of $1 / \lambda$.

(2) Nowhere have we assumed that $\rho$ must have nodes or singular drifts. Even when $\rho$ is always strictly greater than zero, if $\rho(x) \rightarrow 0$ as $|x| \rightarrow \infty$, this 
result may be useful in bounding the probability that $\xi(t)$ ever leaves a sphere of a given radius.

(3) The usual application for bounds such as that of Theorem 1 is to establish the existence and uniqueness of finite-energy diffusions. Note, however, that such bounds are generally useful in taming the singular drifts, which can interfere with efforts to establish even simple assertions analytically. If we can control the probability that the particle ever gets close to the nodes, we can exploit the regularity of the drifts away from the nodes. For an example in which the bound of this paper is used in this manner, see Theorem 5 of [6].

\section{REFERENCES}

1. Ph. Blanchard and S. Golin, Diffusion processes with singular drift fields, Comm. Math. Phys. 109 (1987), 421-435.

2. Eric A. Carlen, Conservative diffusions, Comm. Math. Phys. 94 (1984), 293-315.

3. Existence and sample path properties of the diffusions in Nelson's stochastic mechanics, in Stochastic Processes-Mathematics and Physics, Bielefeld 1984, Springer Lecture Notes in Math. 1158, pp. 25-51.

4. Edward Nelson, Critical diffusions, in Séminaire de Probabilités XIX 1983/84, (J. Azéma and M. Yor, eds.), Springer Lecture Notes in Math. 1123, 1985, pp. 1-11.

5. —_, Quantum fluctuations, Princeton University Press, Princeton, NJ, 1985.

6. Timothy C. Wallstrom, The stochastic mechanics of the Pauli equation, Trans. Amer. Math. Soc. (to appear).

7. W. A. Zheng, Tightness results for laws of diffusion processes. Application to stochastic mechanics, Ann. Inst. H. Poincaré 21 (1985), 103-124.

8. W. A. Zheng and P. A. Meyer, Sur la construction de certaines diffusions, in Séminaire de Probabilités XX, Springer Lecture Notes in Math. 1204, pp. 25-51.

Department of Physics, Princeton University, Princeton, New Jersey 08544

Current address: Department of Physics, California Institute of Technology, Pasadena, California 91125 